\title{
Abrupt onsets do not aid visual search
}

\author{
EILEEN KOWLER \\ Rutgers University, New Brunswick, New Jersey \\ and \\ GEORGE SPERLING \\ New York University, New York, New York
}

\begin{abstract}
In 1980, we showed that visual search with displays containing two abrupt onsets (two brief flashes) was not better than visual search with displays containing one abrupt onset (one long flash). These results showed that saccade-like stimulus transients (abrupt onsets) do not aid visual information processing. This paper extends our previous work by showing that abrupt. and gradual onsets are equally effective for visual search. Subjects searched sequences of displays of alphanumeric characters for a single numeral. Display waveforms were: (1) ramp onset and abrupt offset; (2) abrupt onset and ramp offset; (3) abrupt onset and abrupt offset with duration equal to and (4) half that of the ramp displays. Time between onsets of successive displays was 150 or 250 msec. Performance did not depend on waveform or duration. It was, however, better when the time between onsets of successive displays was $250 \mathrm{msec}$. The results show that visual information processing is unaffected by saccade-like image perturbations. This outcome means that: (1) saccades need be made only to improve the location of the retinal image and not to produce transients, and (2) previously described contributions of abrupt onsets to detection of sinusoidal gratings do not apply to complex information processing tasks.
\end{abstract}

In a previous paper (Kowler \& Sperling, 1980), we found that visual search for a single-numeral target, embedded in an array of letters, was not aided by abrupt onsets. This conclusion was supported by three main results: (1) Visual search with displays containing two abrupt onsets (two brief flashes) was not better than visual search with displays containing a single abrupt onset (one long flash); (2) an abrupt onset created by imposing a rapid, lateral displacement on the stimulus was not useful-visual search with stationary displays was as good as visual search with displays containing a rapid displacement; and (3) visual search was consistent with a model in which information was acquired at a constant rate throughout a stimulus presentation. According to this model, the utility of information acquired long after the onset may decline because redundant information is acquired, and not because the rate of information acquisition slows over time. These three results did not support earlier suggestions that abrupt onsets are required to initiate visual information acquisition (Loftus, 1976; Sperling, Budiansky, Spivak, \& Johnson, 1971). The present paper extends our pre-

This research was supported by Air Force Office of Scientific Research Grants F4960-81-K008 and 82-0085, to E. Kowler, and $82-0279$, to G. Sperling. We thank B. Fischer for serving as a subject, M. Pavel and R. Steinman for useful suggestions, and $\mathbf{C}$. Dellamura for typing the manuscript. E. Kowler's mailing address is: Department of Psychology, Rutgers University, New Brunswick, New Jersey 08903. vious work by showing that gradual onsets are as effective as abrupt onsets for initiating information acquisition.

Understanding the role of abrupt onsets is important, because it has implications for understanding how saccades (high-velocity eye movements) affect visual information acquisition. Saccades produce abrupt changes in the retinal image. If abrupt onsets are helpful, then visual information acquisition would benefit if saccades were made frequently. If abrupt onsets are harmful, then visual information acquisition would benefit if saccades were made as seldom as possible. The relative merits of these two ways of using saccades should be considered, because it is known that human beings can voluntarily choose to adopt either strategy while performing visual tasks (Kowler \& Steinman, 1977; Murphy, 1978; Steinman, Haddad, Skavenski, \& Wyman, 1973). Thus, understanding the role of abrupt onsets may help in understanding the effects of the decisions human beings typically make about saccade frequency, or may lead to suggestions about how saccade frequency could be adjusted to improve visual information acquisition.

\section{THE RATIONALE FOR THE EXPERIMENT}

To examine the effect of abrupt onsets on visual information acquisition, we compared performance in the visual search task we had used earlier (Kowler \& Sperling, 1980) for displays with gradual and with 
abrupt onsets. A brief description of the waveforms of the displays, and the reasons we chose them follows (see Figure 1):

For displays with gradual onsets (ramp-on), intensity increased linearly from zero to a maximum value. As soon as intensity reached the maximum value, the display was abruptly turned off. Fifty milliseconds after the offset, a new ramp-on display appeared. This continued until a sequence of nine consecutive displays, only one of which contained the target numeral, had been presented. A sequence of displays, rather than a single display, was used for two reasons: (1) The sequence permitted control over the time each display was available for processing. Each display was assumed to be available for processing during the interval between its onset and the onset of the next display in the sequence (Sperling, 1963). (2) Using a sequence of displays increased the plausibility of inferences about the role of saccades in visual information processing because a sequence of displays resembles the sequence of foveal views of the world that occurs during the execution of saccades. For this reason, the time between onsets of consecutive displays in the sequence was chosen to be either 150 or 250 msec-typical intersaccadic intervals often reported for reading (Cunitz \& Steinman, 1969; Huey, 1900) or for inspection of pictures (Yarbus, 1967).

Performance with ramp-on displays was compared with performance with two kinds of displays containing abrupt onsets. These displays contained the same amount of energy, integrated over the interval between successive onsets, as was contained in the ramp-on displays. One of these displays-ramp-offhad an abrupt onset and gradual offset. After this display came on, at the same maximum intensity used for the ramp-on displays, the intensity immediately began to decrease linearly to zero. The other displayshort rectangle - had an abrupt onset and abrupt offset. Its intensity was always the same as the maximum value used with the ramp displays. In order for its total energy to be the same as that of the ramp displays, its duration was half that of the ramp displays. Time between successive onsets of the short rectangle, however, was the same as that of the ramp displays. This was achieved by increasing the dark inteval between the offset of a display and the onset of the next display in the sequence.

If abrupt onsets are required for efficient information acquisition, then performance with displays containing abrupt onsets (ramp-off and short rectangle) should be better than performance with the display containing a gradual onset (ramp-on). If abrupt onsets are harmful, then performance with the rampon display should be best.

Comparisons of performance with the different types of displays will also allow inferences about the role of abrupt offsets. For example, if both abrupt onsets and abrupt offsets are helpful, the perfor- mance with the short rectangle should be better than performance with the ramp displays. If both abrupt onsets and abrupt offsets are harmful, then performance with the ramp displays should be better than performance with the short rectangle. This last possible outcome must be interpreted cautiously because better performance with the ramp displays could be due to their greater duration rather than to waveform. A fourth type of stimulus (long rectangle) was included to find out whether stimulus duration affects performance. The duration of this stimulus was twice that of the short rectangle. If performance depends on duration rather than on waveform, then performance with the ramp displays should be better than performance with the short rectangle and the same as performance with the long rectangle. Effects of duration would also be implicated even if performance with the ramps was worse than performance with the long rectangle, because this outcome would occur if the initial portion of the ramp-on display and the final portion of the ramp-off display were too faint to be visually effective.

\section{METHOD}

\section{Stimuli}

Stimuli (similar to those used by Kowler \& Sperling, 1980) were arrays of alphanumeric characters that were displayed on a CRT screen [HP 1240 with a white $(P-4)$ phosphor, controlled by a PDP-11/34 computer]. The screen was located $1.5 \mathrm{~m}$ from the subject. Stimuli were viewed binocularly and a biteboard was used to preserve a fixed distance between the subject's head and the screen.

Arrays contained 24 characters arranged in a $5 \times 5$ square matrix subtending $3.5 \mathrm{deg}$ on a side. The center location of the array always contained the fixation target: two dots separated vertically by $7 \mathrm{~min}$ arc.

A sequence of nine different arrays was presented in each trial. Eight of the arrays contained 24 letters. The remaining array contained 23 letters and a single numeral. The numeral was the target for the search task. Any of the numerals 0 through 9 could be targets. Any letter could appear except B, I, O, Q, and Z, which were excluded because of their resemblance to the numerals 3,1 , 0 , and 2 . The target appeared with equal probability in any of the middle five arrays.

Individual characters subtended $16 \mathrm{~min}$ arc horizontally and $23 \mathrm{~min}$ arc vertically. Their center-to-center separations were $48 \mathrm{~min}$ arc horizontally and $46 \mathrm{~min}$ arc vertically. All characters could be easily identified when the center of the array was fixated.

The characters were illuminated briefly (less than $1 \mathrm{msec}$ ) to a luminous directional energy (LDE) of $0.20 \mathrm{~cd}-\mu \mathrm{sec} /$ point (see Sperling, 1971). Characters contained between 16 and 31 points; thus the LDE/flash for a character ranged between 3.2 and 6.2 cd- $\mu \mathrm{sec} / \mathrm{character}$. These values describe the maximum intensity of characters. Intensity was reduced during gradual onsets and gradual offsets.

The characters were viewed against a $\operatorname{dim}\left(1.3-\mathrm{cd} / \mathrm{m}^{2}\right)$ background that was provided by a flourescent lamp that illuminated the CRT screen. Otherwise, the room was dark.

\section{Procedure}

Pretrial. Before each trial, the subject fixated the two-dot fixation target. When she was ready, she pressed a button that caused the sequence of character arrays to begin $250 \mathrm{msec}$ later.

Waveforms presented during trials. On any trial, all nine character arrays were presented in one of the following ways (see Figure 1): 

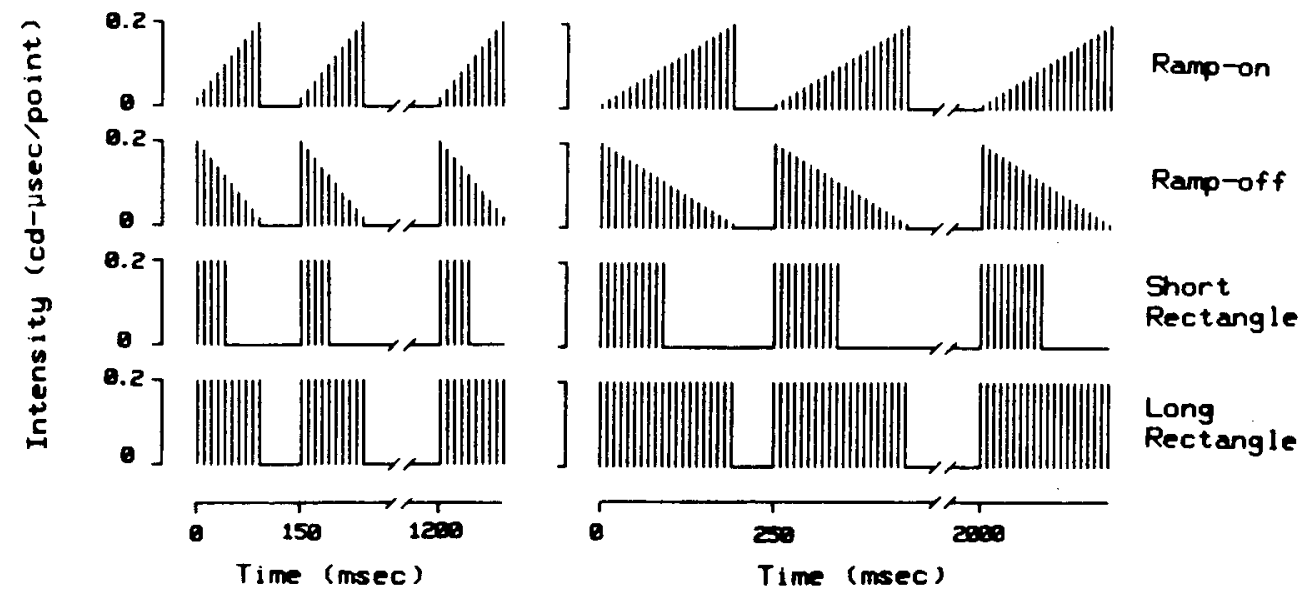

Short

Rectangle

Long

Rectangle

Figure 1. Representation of sequences of arrays during trials with each of the four waveforms when the interval between onsets of successive arrays was 150 msec (left) and 250 msec (right). Each line represents a refresh of the display. The interval between refreshes was 10 msec.

(1) Ramp-on. The intensity of the array increased linearly from zero to the maximum LDE value of $0.20 \mathrm{~cd}-\mu \mathrm{sec} / \mathrm{point}$. Time to reach maximum intensity was either 100 or $200 \mathrm{msec}$. As soon as maximum intensity was reached, the array was turned off. A dark interval of $50 \mathrm{msec}$ intervened between the offset of the array and the onset of the next array in the sequence. Thus, onsets of successive arrays were separated by either 150 or $250 \mathrm{msec}$.

(2) Ramp-off. Same as the ramp-on array except that intensity decreased linearly from the maximum value to zero.

(3) Short rectangle. The intensity of the array was always the maximum value. Arrays were displayed for either 50 or $100 \mathrm{msec}$. Corresponding dark intervals between successive arrays were 100 and $150 \mathrm{msec}$, so that onsets of successive arrays in the sequence were separated by either 150 or $250 \mathrm{msec}$.

(4) Long rectangle. Same as the short rectangle except that arrays were displayed for either 100 or $200 \mathrm{msec}$. Dark intervals were always $50 \mathrm{msec}$ so that onsets of successive arrays in the sequence were separated by 150 or $250 \mathrm{msec}$.

All presentations were actually composed of a series of brief flashes of the array repeated at 10-msec intervals. This refresh interval was short enough so that individual flashes could not be seen and the display appeared continuous. This means that the individual flashes did not constitute separate onsets. We will, therefore, not refer further to the individual flashes, and instead will use the term "waveform" to denote the amplitude envelope of the entire train of flashes. Waveform, unlike the individual flashes, was easily discerned.

Eye movements. Subjects were instructed to refrain from making saccades and to use smooth eye movements to maintain a steady line of sight on the fixation target during the time between the start of the first array and the offset of the ninth array in the sequence. This meant that 1.2 and $2.2 \mathrm{sec}$ of saccade-free fixation were required when the times between successive onsets were 150 and $250 \mathrm{msec}$, respectively. These intervals are short enough so that the occurrence of spontaneous saccades would be rare. ${ }^{1}$

Reports. After each sequence of displays was presented, the subjects had to report (by pressing appropriate buttons) the identity of the target numeral, the location of the target numeral (row and column in the array), and a confidence rating on a 3-point scale for both the identification and location. After each report, they were shown the array that contained the numeral so that they would know the correct answer.

\section{Design}

A mixed-list design was used. The sequence number of the array containing the numeral ( 3 through 7 ), the identity (0 through 9) and the location of the numeral (1 through 24$)$, the waveform (ramp-on, ramp-off, short rectangle, long rectangle), the time between successive onsets (150 or $250 \mathrm{msec}$ ), and the identity and location of the background letters were all chosen randomly, independently, and with replacement. The subject never knew what these choices were before a trial. Each subject was tested in about 2,300 trials distributed over $241 / 2-h$ sessions.

\section{Subjects}

Two subjects were tested: E.K., who was one of the authors, and B.F., who was naive as to the purpose of the experiment. Both were subjects in the Kowler and Sperling (1980) experiment, thus both were highly experienced with the search task. B.F. received no additional practice with the search task before data were collected. E.K.'s additional practice with the search task consisted of serving as a subject in pilot experiments with stimuli similar to those used here.

\section{RESULTS}

Visual search was not improved by abrupt onsets. Performance was virtually the same for all waveforms. This result is summarized in Tables 1 and 2, which show the proportion of trials in which the subjects were correct according to three methods of scoring: (1) correct identification of the numeral, (2) correct location of the numeral, and (3) both correct identification and correct location of the numeral. (Note that these scores are nonindependent and highly correlated measures of performance.) Results are shown separately for the four waveforms and for both the 150- and 250 -msec intervals between onsets of successive arrays. The differences observed among the waveforms were small for all methods of scoring. Statistical tests carried out on the joint identification and location scores showed that differences among waveforms were not significant either for the 150msec interval between onsets of successive arrays $\left[\chi^{2}(3)=3.44, p>.30\right.$, for E.K.; $\chi^{2}(3)=3.64, p>.30$, for B.F.] or for the 250 -msec interval $\left[\chi^{2}(3)=0.27\right.$, p > .95, for E.K.; $\chi^{2}(3)=2.49, p>.50$, for B.F.].

The interval between onsets of successive arrays, unlike waveform, did affect performance no matter which method of scoring was used. Both subjects did 
Table 1

Visual-Search Performance of Subject B.F. for the Four Waveforms and the 150-msec and 250-msec Intervals Between Onsets of Successive Arrays

\begin{tabular}{|c|c|c|c|c|}
\hline Waveform & $\begin{array}{l}\text { Proportion of } \\
\text { Correct } \\
\text { Identifications }\end{array}$ & $\begin{array}{c}\text { Proportion of } \\
\text { Correct } \\
\text { Locations }\end{array}$ & $\begin{array}{l}\text { Proportion of } \\
\text { Correct Identifica- } \\
\text { tions and Locations }\end{array}$ & $\begin{array}{c}\text { Number of } \\
\text { Trials }\end{array}$ \\
\hline \multicolumn{5}{|c|}{150 -msec Interval } \\
\hline $\begin{array}{l}\text { Ramp-on } \\
\text { Ramp-off } \\
\text { Short Rectangle } \\
\text { Long Rectangle }\end{array}$ & $\begin{array}{l}.55 \\
.61 \\
.55 \\
.62\end{array}$ & $\begin{array}{l}.35 \\
.36 \\
.34 \\
.40\end{array}$ & $\begin{array}{l}.31 \\
.34 \\
.29 \\
.37\end{array}$ & $\begin{array}{l}303 \\
303 \\
302 \\
263\end{array}$ \\
\hline All Waveforms & .58 & .36 & .33 & 1171 \\
\hline \multicolumn{5}{|c|}{ 250-msec Interval } \\
\hline $\begin{array}{l}\text { Ramp-on } \\
\text { Ramp-off } \\
\text { Short Rectangle } \\
\text { Long Rectangle }\end{array}$ & $\begin{array}{l}.72 \\
.72 \\
.76 \\
.70\end{array}$ & $\begin{array}{l}.58 \\
.58 \\
.54 \\
.54\end{array}$ & $\begin{array}{l}.56 \\
.56 \\
.52 \\
.52\end{array}$ & $\begin{array}{l}302 \\
302 \\
301 \\
277\end{array}$ \\
\hline All Waveforms & .72 & .56 & .54 & 1182 \\
\hline
\end{tabular}

Table 2

Visual-Search Performance of Subject E.K. for the Four Waveforms and the 150-msec and 250-msec Intervals Between Onsets of Successive Arrays

\begin{tabular}{|c|c|c|c|c|}
\hline Waveform & $\begin{array}{l}\text { Proportion of } \\
\text { Correct } \\
\text { Identifications }\end{array}$ & $\begin{array}{c}\text { Proportion of } \\
\text { Correct } \\
\text { Locations }\end{array}$ & $\begin{array}{l}\text { Proportion of } \\
\text { Correct Identifica- } \\
\text { tions and Locations }\end{array}$ & $\begin{array}{c}\text { Number of } \\
\text { Trials }\end{array}$ \\
\hline \multicolumn{5}{|c|}{150 -msec Interval } \\
\hline $\begin{array}{l}\text { Ramp-on } \\
\text { Ramp-off } \\
\text { Short Rectangle } \\
\text { Long Rectangle }\end{array}$ & $\begin{array}{l}.68 \\
.61 \\
.63 \\
.67\end{array}$ & $\begin{array}{l}.49 \\
.48 \\
.47 \\
.52\end{array}$ & $\begin{array}{l}.46 \\
.44 \\
.43 \\
.50\end{array}$ & $\begin{array}{l}277 \\
311 \\
303 \\
265\end{array}$ \\
\hline All Waveforms & .64 & .49 & .46 & 1156 \\
\hline \multicolumn{5}{|c|}{ 250-msec Interval } \\
\hline $\begin{array}{l}\text { Ramp-on } \\
\text { Ramp-off } \\
\text { Short Rectangle } \\
\text { Long Rectangle }\end{array}$ & $\begin{array}{l}.74 \\
.73 \\
.72 \\
.65\end{array}$ & $\begin{array}{l}.62 \\
.63 \\
.61 \\
.60\end{array}$ & $\begin{array}{l}.60 \\
.61 \\
.59 \\
.58\end{array}$ & $\begin{array}{l}310 \\
285 \\
314 \\
294\end{array}$ \\
\hline All Waveforms & .71 & .62 & .59 & 1203 \\
\hline
\end{tabular}

substantially and significantly better with the 250msec interval $\left[\chi^{2}(1)=44.8, p<.001\right.$, for E.K.; $\chi^{2}(1)=$ $106.7, \mathrm{p}<.001$, for B.F.; see Tables 1 and 2].

Visual search also depended on the location and the identity of the target numerals. Some targets (e.g., a 7 located to the left of the fixation target) were easy to find, while others (e.g., a 5 located in the bottom row) were difficult. Nevertheless, performance across the varying levels of stimulus difficulty was determined by the time between successive onsets rather than by waveform.

\section{DISCUSSION}

Our results show that abrupt onsets are not required to initiate visual information acquisition. These results extend our prior findings (Kowler \& Sperling, 1980). Previously, we showed that adding a second abrupt onset was neither useful nor necessary for maintaining visual information acquisition once it was underway. We have now shown that even the first abrupt onset accompanying the initial view of the display is not required to initiate visual information acquisition. Specifically, we found that performance with waveforms containing abrupt onsets was the same as performance with waveforms containing gradual onsets. Similarly, neither the type of offset nor the duration of the display made any difference. Performance was affected only by the interval between onsets of successive arrays. Thus, success in visual search depended on the total amount of time available to process displays rather than on how the time was apportioned between stimulus and dark intervals.

\section{Comparison With Other Studies of Abrupt \\ Onsets and Visual Information Acquisition}

Previous investigators have also reported that visual information acquisition depends on the total time available to process displays rather than on how 
the time is apportioned between stimulus and dark intervals (Haber \& Nathanson, 1969; Sperling \& Melchner, 1978). These results had been thought to support an important role for abrupt onsets (Sperling \& Melchner, 1978). According to such an interpretation, it is the initial abrupt onset, shared by all displays regardless of their duration, which initiates visual information acquisition. The continued presence of a display after the abrupt onset is not useful. This interpretation could explain previous reports of equivalent performance for different duration displays, because the previous stimuli always contained an abrupt onset. But it cannot explain our results, because we found that gradual onsets were as effective as abrupt onsets for initiating visual information acquisition. Thus, prior findings (Haber \& Nathanson, 1969; Sperling \& Melchner, 1978) that brief- and long-duration displays produce the same performance need not imply that abrupt onsets are important. Instead, such findings imply that memory for information contained in a brief flash can be useful for 100 to $200 \mathrm{msec}$ after the flash is turned off. This interpretation agrees with Kowler and Sperling's (1980) findings that performance with long flashes is the same as performance with brief flashes provided that the difference in the durations is less than about $\mathbf{2 0 0}$ msec. Further implications of findings that brief and long flashes can produce the same performance are described in the next section.

\section{Comparison With Studies of Abrupt Onsets and Contrast Sensitivity: Energy-Limited vs. Time-Limited Processes}

Stimulus waveforms similar to those we used were employed by Breitmeyer and Julesz (1975). They measured the minimum contrast required for detection of sinusoidal gratings presented either with abrupt or with gradual (ramp) onsets and offsets. They found that the type of offset never affected contrast sensitivity. The type of onset affected contrast sensitivity only when the spatial frequency of the grating was less than about 5 cycles/deg. Detection of these gratings was improved by abrupt onsets. Detection of gratings of higher spatial frequency was unaffected by onset waveform (see Tolhurst, 1975, for similar results). These results are consistent with suggestions that gratings are detected by independent visual channels that have correlated spatial and temporal parameters (Watson \& Nachmias, 1977), that is, some channels are maximally sensitive to high spatial frequencies and low temporal frequencies, and others are sensitive to low spatial frequencies and high temporal frequencies.

On the surface, our present and prior (Kowler \& Sperling, 1980) findings that abrupt onsets do not aid visual search appear to be similar to the previous findings, summarized above, that abrupt onsets do not aid detection of high spatial frequency gratings.
Further consideration of the detection and search tasks, however, suggests that comparison of the two sets of results is misleading for the following reasons.

Detection of sinusoidal gratings at threshold is an energy-limited process. This means that detection will be best when all the stimulus energy is packed in a very brief flash. Detection gets worse as energy is spread over time. The studies of detection of sinusoidal gratings described above attempt to characterize how detection changes as a function of the spread of stimulus energy over time. ${ }^{2}$

Our study of visual search is not like studies of detection of sinusoidal gratings, because all of our stimuli contained sufficient energy to be easily detectable. Thus, we are attempting to characterize processes that occur after detection, for example, resolution and recognition of details within each one of many characters so that decisions about whether a character is a numeral can be made. Our results show that these processes are not energy-limited. Rather, they are time-limited. This means that performance is determined by the total time in which stimuli are available, rather than by total stimulus energy (Sperling, 1979). The visual search task we used satisfies this definition because we found that performance depended on the time between onsets of successive arrays and not on stimulus energy or waveform. Specifically, we found that increasing the time available to process displays by adding an interval of darkness after an array was turned off was helpful. On the other hand, increasing stimulus energy by leaving an array on longer before the next array appeared was not helpful. Similarly, redistributing the energy in the array so that most energy occurred near the onset (in ramp-off displays) or near the offset (in ramp-on displays) was not helpful. Finding that performance depended on time available to process displays rather than on energy is consistent with prior findings that visual search with a brief flash followed by darkness is the same as visual search with a long flash provided that the difference in duration of the brief and long flash does not exceed about 200 msec (Kowler \& Sperling, 1980; Sperling \& Melchner, 1978).

To summarize, our results suggest that visual search is a time-limited process. This means that stimulus energy or waveform, parameters which affect energylimited tasks, such as detection, do not affect visual search once stimuli are detectable. These considerations suggest that it is not possible to make inferences about complex processes, such as those involved in visual search, from studies of detection of targets at threshold. This conclusion may not be limited to visual search. Others have shown that hyperacuity tasks (Westheimer, 1979), counting and reading (Sperling, 1979), and partial-report tasks (Adelson \& Jonides, 1980) are not improved by increasing stimulus energy; that is, these tasks also are to some extent time-limited rather than energy-limited. 


\section{Implications for Saccades}

Saccades perturb the retinal image. Specifically, they produce rapid displacements which lead to the abrupt offset of an image at one retinal location and the abrupt onset of an image at a new retinal location. Our present and prior results (Kowler \& Sperling, 1980) show that imposing such saccade-like perturbations on a stimulus does not affect visual search. This suggests that retinal image perturbations created in natural situations by saccades neither help nor harm visual information acquisition.

The failure of retinal image perturbations produced by saccades to affect visual information acquisition is a useful result for the following reason. It is known that saccades are not reflexive eye movements whose characteristics are automatically determined by physical properties of the stimulus, such as stimulus size, color, shape, or luminance (Murphy, Haddad, \& Steinman, 1974; Steinman, 1965, 1976; Winterson \& Steinman, 1978). Instead, human beings can voluntarily choose to look when and where they wish (Steinman et al., 1973). One consideration likely to influence these choices is the effect of saccades on visual information acquisition. If the retinal image perturbations created by saccades were helpful for visual information acquisition, then saccades should be made frequently, even if this decision means that the line of sight may sometimes be taken away from details of interest. If, on the other hand, the perturbations were harmful to visual information acquisition, then saccades should be made infrequently and the line of sight kept in one place while attention is directed as far into the peripheral portion of the visual field as possible. Our results suggest that neither of these two strategies for using saccades need be adopted, because the retinal image perturbations produced by saccades do not affect visual information acquisition. Such a result is useful because it reduces the number of factors that must be taken into account when deciding when and where to make saccades. Perhaps only the need to bring images of selected details onto retinal locations that are best suited for visual processing need be considered in planning saccades.

The suggestion that saccades are required only to bring images onto retinal locations best suited for visual processing, and not to create abrupt onsets, is consistent with what is known about the effect of saccades on the performance of visual tasks. Studies have shown that increasing the frequency of saccades can improve performance of such visual tasks as counting (Kowler \& Steinman, 1977) and picture recognition (Loftus, 1972). Prior interpretations of such results had suggested that saccades were useful because they created abrupt onsets which were necessary for efficient visual information acquisition (Kowler \& Sperling, 1980; Loftus, 1976). This interpretation seemed particularly attractive for explain- ing benefits derived from saccades which were not needed to improve visual acuity by bringing retinal images into the fovea. For example, Kowler and Steinman (1977) found that counting accuracy for items haphazardly arranged in a small, foveal (2-degdiam) display was higher when saccades were used to count than when saccades were not used and the line of sight was maintained at the center of displays. Saccades were not needed to improve visual acuity in that experiment because all items could be clearly distinguished when the line of sight remained at the center of the small displays. Our present and prior (Kowler \& Sperling, 1980) results suggest that the reason saccades were useful for counting was not that visual information acquisition was improved by the abrupt onsets created by saccades. Alternative explanations for the observed benefits of saccades, which do not involve abrupt onsets, are required.

One plausible reason that saccades were useful for counting was that saccades brought images into a small, central, retinal region within the fovea in which some aspect of visual processing, other than visual acuity, is best. This speculation is supported by findings that saccades that are smaller than about $\mathbf{3 0} \mathrm{min}$ arc are useless for performance of visual tasks (Kowler \& Steinman, 1980). For example, small saccades do not aid counting (Kowler \& Steinman, 1979), and nor do they aid the performance of finely guided visuomotor tasks (Winterson \& Collewijn, 1976) or visual acuity tasks (Bridgeman \& Palca, 1980). Thus, evidence from studies of the effects of eye movements on visual information processing is consistent with our results: both suggest that saccades may be required only to bring images into a retinal region that is optimal for visual processing. Abrupt onsets are useless.

\section{REFERENCES}

Adelson, E. H., \& Jonides, J. The psychophysics of iconic storage. Journal of Experimental Psychology: Human Perception and Performance, 1980, 6, 486-493.

Breitmeyer, B., \& Julesz, B. The role of on and off transients in determining the psychophysical spatial frequency response. Vision Research, 1975, 15, 411-415.

Bridgeman, B., \& Palca, J. The role of microsaccades in high acuity observational tasks. Vision Research, 1980, 20, 813-817.

Ciuffreda, K. J., Kenyon, R. V., \& Stark, L. Suppression of fixational saccades in strabismic and anisometropic amblyopia. Ophthalmic Research, 1979, 11, 31-39.

Cunitz, R. J., \& Ste inman, R. M. Comparison of saccadic eye movements during fixation and reading. Vision Research, 1969, 9, 683-693.

Haber, R. N., \& Nathanson, L. S. Processing of sequentially presented letters. Perception \& Psychophysics, 1969, 5, 359-361.

Haddad, G. M., \& Steinman, R. M. The smallest voluntary saccade: Implications for fixation. Vision Research, 1973, 13, 1075-1086.

Haddad, G. M., \& Winterson, B. J. Effect of flicker on oculomotor performance. In G. Lennerstrand \& P. Bach-y-Rita (Eds.), Basic mechanisms of ocular motility and their clinical implications. Oxford: Pergamon, 1975.

HUEY, E. B. On the psychology and physiology of reading. American Journal of Psychology, 1900, 11, 283-302. 
Kowler, E., \& Sperling, G. Transient stimulation does not aid visual search: Implications for the role of saccades. Perception \& Psychophysics, 1980, 27, 1-10.

Kowlen, E., \& Steinman, R. M. The role of small saccades in counting. Vision Research, 1977, 17, 141-146.

Kowler, E., \& Steinman, R. M. Miniature saccades: Eye movements that do not count. Vision Research, 1979, 19, 105-108.

Kowler, E., \& Ste inman, R. M. Small saccades serve no useful purpose. Vision Research, 1980, 20, 273-276.

Loftus, G. Eye fixations and recognition memory for pictures. Cognitive Psychology, 1972, 3, 525-551.

LofTus, G. A framework for a theory of picture recognition. In R. A. Monty \& J. W. Senders (Eds.), Eye movements and psychological processes. Hillsdale, N.J: Erlbaum, 1976.

Murphy, B. J. Pattern thresholds for moving and stationary gratings. Vision Research, 1978, 18, 521-530.

Murphy, B. J., Haddad, G. M., \& Steinman, R. M. Simple forms and fluctuations in the line of sight: Implications for motor theories of form processing. Perception \& Psychophysics, 1974, 16, 557-563.

Munphy, B. J., Kowlen, E., \& Steinman, R. M. Slow oculomotor control in the presence of moving backgrounds. Vision Research, 1975, 15, 1263-1268.

Schor, C., \& Hallmark, W. Slow control of eye position in strabismic amblyopia. Investigative Ophthalmology, 1978, 17, 577-581.

Sperling, G. A model for visual memory tasks. Human Factors, $1963,5,19-31$.

SPERLING, G. The description and luminous calibration of cathode ray oscilloscope visual displays. Behavior Research Methods \& Instrumentation, 1971, 3, 148-151.

Sperling, G. Critical duration, supersummation, and the narrow domain of strength-duration experiments. The Behavioral and Brain Sciences, 1979, 2, 279-281.

Sperling, G., Budiangky, J., Spivak, J. G., \& Johngon, M. C. Extremely rapid visual search: The maximum rate of scanning letters for the presence of a numeral. Science, 1971, 174, 307-311.

Sperling, G., \& Melchner, M. J. Visual search, visual attention and the attention operating characteristic. In $\mathrm{J}$. Requin (Ed.), Attention and performance VII. Hillsdale, N.J: Erlbaum, 1978.

Stermman, R. M. Effect of target size, luminance, and color on monocular fixation. Journal of the Optical Society of America, $1965,55,1158-1165$.

Ste inman, R. M. The role of eye movements in maintaining a phenomenally clear and stable world. In R. A. Monty \& J. M. Senders (Eds.), Eye movements and psychological processes. Hillsdale, N.J: Erlbaum, 1976.

Steinman, R. M., Cunitz, R. J., Timberlake, G. T., \& HermaN, M. Voluntary control of microsaccades during maintained monocular fixation. Science, 1967, 155, 1577-1579.
Steinman, R. M., Hadpad, G. M., Skavenski, A. A., \& Wyman, D. Miniature eye movement. Science, 1973, 181, 810-819.

Tolhurst, D. J. Reaction times in the detection of gratings by human observers: A probabilistic mechanism. Vision Research, $1975,15,1143-1149$.

Watson, A. B., \& Nachmias, J. Patterns of temporal interaction in the detection of gratings. Vision Research, 1977, 17, 893-902.

WESTHEIMER, G. The spatial sense of the eye. Investigative Ophthalmology and Visual Science, 1979, 18, 893-912.

Winterson, B. J., \& CollewiJn, H. Microsaccades during finely guided visuomotor tasks. Vision Research, 1976, 16, 1387-1390.

Winterson, B. J., \& Steinman, R. M. The effect of luminance on human smooth pursuit of perifoveal and foveal targets. Vision Research, 1978, 18, 1165-1172.

Yarbus, A. L. Eye movements and vision. New York: Plenum Press, 1967.

\section{NOTES}

1. It is well known that knowledgeable subjects experienced in eye-movement experiments, as well as naive, inexperienced subjects, can refrain from making saccades and use smooth eye movements exclusively to maintain the line of sight for many seconds after simple verbal instructions (Haddad \& Steinman, 1973; Steinman, Cunitz, Timberlake, \& Herman, 1967; Winterson \& Collewijn, 1976). The same results have been obtained for naive subjects with amblyopia, who can refrain from making saccades for many seconds after verbal instructions despite the presence of the high smooth eye-movement velocities characteristic of amblyopia (Ciuffreda, Kenyon, \& Stark, 1979; Schor \& Hallmark, 1978). Subjects can also refrain from making saccades for many seconds with many different kinds of stimuli, for example, simple forms (Murphy et al., 1974), large flickering fields (Haddad \& Winterson, 1975), and moving backgrounds (Murphy, Kowler, \& Steinman, 1975), and while performing visual, cognitive, and visuomotor tasks, for example, making contrast threshold judgments about a moving sinusoidal grating (Murphy, 1978), counting visual items (Kowler \& Steinman, 1977, 1979), and threading a needle and aiming and shooting a rifle (Winterson \& Collewijn, 1976). (See also Kowler \& Steinman, 1980, and Steinman et al., 1973 , for further reviews of this material.)

2. Technically, the temporal waveform of a sinusoidal grating determines the spectral power density of the stimulus over timewhat may be called "Fourier energy"-rather than total stimulus energy. Our discussion of energy-limited processes assumes that Fourier energy and stimulus energy are detected in analogous ways by the visual system.

Manuscript received January 26, 1983; revision accepted for publication April 5, 1983.) 